\title{
Exact solutions to linear and nonlinear wave and diffusion equations
}

\author{
M. A. AL-Jawary \\ Head of Department of Mathematics, College of Education for Pure Sciences / Ibn-AL-Haithem, Baghdad University, Baghdad, Iraq \\ E-mail: Majeed.a.w@ihcoedu.uobaghdad.edu.iq
}

Copyright (C)2015 M. A. AL-Jawary. This is an open access article distributed under the Creative Commons Attribution License, which permits unrestricted use, distribution, and reproduction in any medium, provided the original work is properly cited.

\begin{abstract}
In the present paper, the new iterative method proposed by Daftardar-Gejji and Jafari (NIM or DJM) [V. DaftardarGejji, H. Jafari, An iterative method for solving non linear functional equations, J. Math. Anal. Appl. 316 (2006) 753-763] is used for solving linear and nonlinear wave and diffusion equations. In this iterative method the solution is obtained in the series form that converge to the exact solution with easily computed components. The results demonstrate that the method has many merits such as being derivative-free, overcome the difficulty arising in calculating Adomian polynomials to handle the nonlinear terms in Adomian Decomposition Method (ADM). It does not require to calculate Lagrange multiplier as in Variational Iteration Method (VIM) and no needs to construct a homotopy and solve the corresponding algebraic equations as in Homotopy Perturbation Method (HPM). A comparison with some existing techniques such as ADM, HPM and VIM also presented, which shows that the DJM is effective and convenient to use and overcomes the difficulties arising in existing techniques. The results show that the present method is very effective and simple and provide the analytic solutions. The software used for the calculations in this study was MATHEMATICA ${ }^{\circledR}$ 8.0.
\end{abstract}

Keywords: Diffusion equations, Exact solution, New iterative method, Wave equations

\section{Introduction}

A variety of problems in physics, chemistry and biology have their mathematical setting as linear and nonlinear ordinary or partial differential equations. Many methods have been developed to solve differential equations, especially nonlinear, which are receiving increasing attention.

Many of the phenomena that arise in mathematical physics and engineering fields can be described by partial differential equations (PDEs). In physics for example, the heat flow and the wave propagation phenomena are well described by partial differential equations. Moreover, most physical phenomena of fluid dynamics, quantum mechanics, electricity, plasma physics, propagation of shallow water waves, and many other models are formulated by partial differential equations [1].

Due to these huge applications, there is a demand on the development of accurate and efficient analytic or approximate methods able to deal with the PDEs.

Many fields of science, engineering and physical problems can be described by initial boundary value problems (IBVP). Except for a few number of these problems, we encounter difficulties in finding their exact analytical solutions. 
Many attempts have been made to develop analytic and approximate methods to solve the linear and nonlinear wave and diffusion equations, see $[2,3,4,5]$. Although such methods have been successfully applied but some difficulties have appeared, for examples, in calculating Adomian polynomials to handle the nonlinear terms in ADM [2], calculate Lagrange multiplier in VIM [3, 4, 5], construct a homotopy and solve the corresponding algebraic equations in HPM [5].

Recently, Daftardar-Gejji and Jafari [6] have proposed a new technique for solving linear/nonlinear functional equations namely new iterative method (NIM) or (DJM). The DJM has been extensively used by many researchers for the treatment of linear and nonlinear ordinary and partial differential equations of integer and fractional order, see $[7,8,9,10,11,12,13,14,15,16]$. The method converges to the exact solution if it exists through successive approximations. However, for concrete problems, a few approximations can be used for numerical purposes with high degree of accuracy. The DJM is simple to understand and easy to implement using computer packages and yields better results and does not require any restrictive assumptions for nonlinear terms as required by some existing techniques.

In this paper, the applications of the DJM for the linear and nonlinear wave and diffusion equations will be presented. For wave equations, the analytic solutions are obtained for problems in bounded and unbounded domains. Also, for diffusion problems the exact solutions are developed for some diffusion processes of power law diffusitivies.

The results obtained in this paper are compared with those obtained by other iterative methods such as ADM [2], VIM [3, 4, 5], and HPM [5]. Comparisons show that the DJM is effective and convenient to use and overcomes the difficulties arising in others existing techniques.

The present paper has been organized as follows. In section 2 is devoted to the description the new iterative method (NIM or DJM) and its convergence. In section 3 the linear and nonlinear wave and diffusion equations are solved by DJM and finally in section 4 the conclusion is presented.

\section{The new iterative method (NIM or DJM)}

Consider the following general functional equation:

$u=N(u)+f$,

where $N$ is a nonlinear operator from a Banach space $B \rightarrow B$ and $f$ is a known function[6, 7, 8, 9, 10]. We are looking for a solution $u$ of Eq.(1) having the series form:

$u=\sum_{i=0}^{\infty} u_{i}$.

The nonlinear operator $N$ can be decomposed as

$N\left(\sum_{i=0}^{\infty} u_{i}\right)=N\left(u_{0}\right)+\sum_{i=1}^{\infty}\left\{N\left(\sum_{j=0}^{i} u_{j}\right)-N\left(\sum_{j=0}^{i-1} u_{j}\right)\right\}$.

From Eqs.(2) and (3), Eq.(1) is equivalent to

$\sum_{i=0}^{\infty} u_{i}=f+N\left(u_{0}\right)+\sum_{i=1}^{\infty}\left\{N\left(\sum_{j=0}^{i} u_{j}\right)-N\left(\sum_{j=0}^{i-1} u_{j}\right)\right\}$

We define the recurrence relation:

$$
\begin{aligned}
& G_{0}=u_{0}=f \\
& G_{1}=u_{1}=N\left(u_{0}\right), \\
& G_{m}=u_{m+1}=N\left(u_{0}+\cdots+u_{m}\right)-N\left(u_{0}+\cdots+u_{m-1}\right), \quad m=1,2, \ldots
\end{aligned}
$$

Then

$\left(u_{1}+\ldots+u_{m+1}\right)=N\left(u_{1}+\ldots+u_{m}\right), \quad m=1,2, \ldots$, 
and

$u(x)=f+\sum_{i=1}^{\infty} u_{i}$

The m-term approximate solution of Eq.(2) is given by $u=u_{0}+u_{1}+\ldots+u_{m-1}$.

\subsection{Convergence of the DJM}

We present below the condition for convergence of the series $\sum u_{i}$. For more details we refer the reader to [17].

Theorem 3.1.1:[17]

If $\mathrm{N}$ is $C^{(\infty)}$ in a neighbourhood of $u_{0}$ and $\left\|N^{(n)}\left(u_{0}\right)\right\| \leq L$, for any $n$ and for some real $L>0$ and $\left\|u_{i}\right\| \leq M<\frac{1}{e}$,

$i=1,2, \ldots$, then the series $\sum_{n=0}^{\infty} G_{n}$ is absolutely convergent and moreover, $\left\|G_{n}\right\| \leq L M^{n} e^{n-1}(e-1), n=1,2, \ldots$

Theorem 3.1.2:[17]

If $N$ is $C^{(\infty)}$ and $\left\|N^{(n)}\left(u_{0}\right)\right\| \leq M \leq e^{-1}, \forall n$, then the series $\sum_{n=0}^{\infty} G_{n}$ is absolutely convergent.

\section{Solution of wave and diffusion equations by using DJM}

In this section the DJM will be applied to solve the linear and nonlinear wave and diffusion equations independently.

\subsection{Wave equations}

The linear and nonlinear wave equations in bounded and in an unbounded domains are given by [3]:

$u_{t t}=u_{x x}+f(u), \quad 0<x<L, \quad t>0$

$u_{t t}=u_{x x}+F(u)+g(x, t), \quad 0<x<L, \quad t>0$

$u_{t t}=\frac{x^{2}}{2} u_{x x}, \quad 0<x<L, \quad t>0$

$u_{t t}=u_{x x}, \quad-\infty<x<\infty, \quad t>0$

respectively. The functions $f(u), F(u)$ and $g(x, t)$ are linear, nonlinear and source functions, respectively. The wave equation plays an important role in various physical problems. Study of the wave equation is needed in diverse areas of engineering and scientific applications [3].

The DJM will be applied to four physical models to illustrate the strength of the method and to obtain exact solutions for these models. 


\subsubsection{The homogeneous wave equation}

Let us consider the homogeneous wave equation [3]:

$u_{t t}=u_{x x}-3 u, 0<x<\pi, t>0$,

with initial condition:

$u(x, 0)=0, \quad u_{t}(x, 0)=2 \cos x$,

and the boundary conditions

$u(0, t)=\sin 2 t, \quad u(\pi, t)=-\sin 2 t$.

It is worth to mention that an additional term $-3 u$ is added to the standard wave equation. This term arises when each element of the string is subject to an additional force which is proportional to its displacement.

Eq.(15) can be written in an operator form as

$L_{t t} u=u_{x x}-3 u$,

where $L_{t t}=\frac{\partial^{2}}{\partial t^{2}}$. Let us assume the the inverse operator $L_{t t}^{-1}$ exists and it can be take with respect $t$ from 0 to $t$, i.e.

$L_{t t}^{-1}()=.\int_{0}^{t} \int_{0}^{t}() d t d$.

Then, by taking the inverse operator $L_{t t}^{-1}$ to both sides of the Eq.(15) and using the initial condition, leads to

$u(x, t)=2 t \cos x+L_{t t}^{-1}\left(u_{x x}-3 u\right)$.

By applying the DJM for Eq.(17) the following recurrence relation for the determination of the components $u_{n+1}(x, t)$ are obtained:

$u_{0}(x, t)=2 t \cos x$

$u_{1}(x, t)=N\left(u_{0}\right)=L_{t t}^{-1}\left(\left(u_{0}\right)_{x x}-3 u_{0}\right)=-\frac{4}{3} t^{3} \cos x=-\frac{(2 t)^{3}}{3 !} \cos x$,

$u_{2}(x, t)=N\left(u_{1}+u_{0}\right)-N\left(u_{0}\right)=L_{t t}^{-1}\left(\left(u_{1}+u_{0}\right)_{x x}-3\left(u_{1}+u_{0}\right)\right)-u_{1}=\frac{4}{15} t^{5} \cos x=\frac{(2 t)^{5}}{5 !} \cos x$,

$u_{3}(x, t)=N\left(u_{2}+u_{1}+u_{0}\right)-N\left(u_{1}+u_{0}\right)=L_{t t}^{-1}\left(\left(u_{2}+u_{1}+u_{0}\right)_{x x}-3\left(u_{2}+u_{1}+u_{0}\right)\right)-$

$$
-L_{t t}^{-1}\left(\left(u_{1}+u_{0}\right)_{x x}-3\left(u_{1}+u_{0}\right)\right)=-\frac{2}{315} t^{7} \cos x=-\frac{(2 t)^{7}}{7 !} \cos x,
$$

and so on.

Continuing in this manner, the $(n+1)$ th approximation of the exact solutions for the unknown functions $u(x, t)$ can be achieved as: 


$$
\begin{aligned}
u_{n+1}=N\left(u_{0}+\cdots+u_{n}\right)-N\left(u_{0}\right. & \left.+\cdots+u_{n-1}\right)=L_{t t}^{-1}\left(\left(u_{0}+\cdots+u_{n}\right)_{x x}-3\left(u_{0}+\cdots+u_{n}\right)\right)- \\
& -L_{t t}^{-1}\left(\left(u_{0}+\cdots+u_{n-1}\right)_{x x}-3\left(u_{0}+\cdots+u_{n-1}\right)\right), \quad n=1,2, \cdots
\end{aligned}
$$

Based on the DJM, we constructed the solution $u(x, t)$ as:

$u(x, t)=\sum_{k=0}^{n} u_{k}(x, t) \quad n \geq 0$.

Therefore, according to Eq.(23), we get:

This has the closed form

$$
u_{n}(x, t)=\cos x\left((2 t)-\frac{(2 t)^{3}}{3 !}+\frac{(2 t)^{5}}{5 !}-\frac{(2 t)^{7}}{7 !}+\cdots\right)
$$

$u(x, t)=\cos x \sin 2 t$.

which is the exact solution of the problem and it is the same results obtained by VIM [3].

\subsubsection{The inhomogeneous wave equation}

We next consider the inhomogeneous nonlinear wave equation [3]:

$u_{t t}=u_{x x}+u+u^{2}-x t-x t^{2}, 0<x<\pi, t>0$,

with initial condition:

$u(x, 0)=0, \quad u_{t}(x, 0)=x$,

and the boundary conditions

$u(0, t)=0, u(\pi, t)=\pi t$.

According to the DJM, we achieve the following components:

$u_{0}(x, t)=x t$,

$u_{1}(x, t)=N\left(u_{0}\right)=L_{t t}^{-1}\left(\left(u_{0}\right)_{x x}+\left(u_{0}\right)+\left(u_{0}\right)^{2}-x t-x t^{2}\right)=0$,

$u_{2}(x, t)=N\left(u_{1}+u_{0}\right)-N\left(u_{0}\right)=L_{t t}^{-1}\left(\left(u_{1}+u_{0}\right)_{x x}+\left(u_{1}+u_{0}\right)+\left(u_{1}+u_{0}\right)^{2}-x t-x t^{2}\right)-u_{1}=0$,

$u_{3}(x, t)=N\left(u_{2}+u_{1}+u_{0}\right)-N\left(u_{1}+u_{0}\right)=L_{t t}^{-1}\left(\left(u_{2}+u_{1}+u_{0}\right)_{x x}+\left(u_{2}+u_{1}+u_{0}\right)+\left(u_{2}+\right.\right.$

$$
\left.\left.+u_{1}+u_{0}\right)^{2}-x t-x t^{2}\right)-L_{t t}^{-1}\left(\left(u_{1}+u_{0}\right)_{x x}+\left(u_{1}+u_{0}\right)+\left(u_{1}+u_{0}\right)^{2}-x t-x t^{2}\right)=0,
$$

and so on. In fact, we have $u_{n}(x, t)=0$, for $n \geq 1$.

Therefore, according to Eq.(23), we get:

$u(x, t)=x t$.

which is the exact solution of the problem and it is the same results obtained by VIM [3]. 


\subsubsection{The wave-like equation}

We now consider the wave-like equation [3]:

$u_{t t}=\frac{x^{2}}{2} u_{x x}, \quad 0<x<1, \quad t>0$,

with initial condition:

$u(x, 0)=0, u_{t}(x, 0)=x^{2}$,

and the boundary conditions

$u(0, t)=0, u(\pi, t)=\sinh t$.

By applying the DJM, the following recurrence relation for the determination of the components $u_{n+1}(x, t)$ are obtained:

$u_{0}(x, t)=x^{2} t$

$u_{1}(x, t)=N\left(u_{0}\right)=L_{t t}^{-1}\left(\frac{x^{2}}{2}\left(u_{0}\right)_{x x}\right)=x^{2} \frac{t^{3}}{3 !}$,

$u_{2}(x, t)=N\left(u_{1}+u_{0}\right)-N\left(u_{0}\right)=L_{t t}^{-1}\left(\frac{x^{2}}{2}\left(u_{1}+u_{0}\right)_{x x}\right)-u_{1}=x^{2} \frac{t^{5}}{5 !}$,

$u_{3}(x, t)=N\left(u_{2}+u_{1}+u_{0}\right)-N\left(u_{1}+u_{0}\right)=L_{t t}^{-1}\left(\frac{x^{2}}{2}\left(u_{2}+u_{1}+u_{0}\right)_{x x}\right)-L_{t t}^{-1}\left(\frac{x^{2}}{2}\left(u_{1}+u_{0}\right)_{x x}\right)=x^{2} \frac{t^{7}}{7 !}$,

and so on.

Continuing in this manner, the $(n+1)$ th approximation of the exact solutions for the unknown functions $u(x, t)$ can be achieved as:

$$
\begin{array}{r}
u_{n+1}=N\left(u_{0}+\cdots+u_{n}\right)-N\left(u_{0}+\cdots+u_{n-1}\right)=L_{t t}^{-1}\left(\frac{x^{2}}{2}\left(u_{0}+\cdots+u_{n}\right)_{x x}\right)- \\
-L_{t t}^{-1}\left(\frac{x^{2}}{2}\left(u_{0}+\cdots+u_{n-1}\right)_{x x}\right), \quad n=1,2, \ldots
\end{array}
$$

Therefore, according to Eq.(23), we get:

$u(x, t)=x^{2}\left(t+\frac{t^{3}}{3 !}+\frac{t^{5}}{5 !}+\frac{t^{7}}{7 !}+\cdots\right)$

This has the closed form

$u(x, t)=x^{2} \sinh t$.

which is the exact solution of the problem and it is the same results obtained by VIM [3]. 


\subsubsection{The wave equation in unbounded domain}

We consider the wave equation in an unbounded domain [3]:

$u_{t t}=u_{x x}, \quad-\infty<x<\infty, \quad t>0$

with initial condition:

$u(x, 0)=\sin x, u_{t}(x, 0)=0$,

Proceeding as before, the recurrence relation

$u_{0}(x, t)=\sin x$,

$u_{1}(x, t)=N\left(u_{0}\right)=L_{t t}^{-1}\left(\left(u_{0}\right)_{x x}\right)=-\frac{t^{2}}{2 !}$,

$u_{2}(x, t)=N\left(u_{1}+u_{0}\right)-N\left(u_{0}\right)=L_{t t}^{-1}\left(\left(u_{1}+u_{0}\right)_{x x}\right)-u_{1}=\frac{t^{4}}{4 !}$

$u_{3}(x, t)=N\left(u_{2}+u_{1}+u_{0}\right)-N\left(u_{1}+u_{0}\right)=L_{t t}^{-1}\left(\left(u_{2}+u_{1}+u_{0}\right)_{x x}\right)-L_{t t}^{-1}\left(\left(u_{1}+u_{0}\right)_{x x}\right)=-\frac{t^{6}}{6 !}$,

and so on.

Continuing in this manner, the $(n+1)$ th approximation of the exact solutions for the unknown functions $u(x, t)$ can be achieved as:

$$
\begin{array}{r}
u_{n+1}=N\left(u_{0}+\cdots+u_{n}\right)-N\left(u_{0}+\cdots+u_{n-1}\right)=L_{t t}^{-1}\left(\left(u_{0}+\cdots+u_{n}\right)_{x x}\right)- \\
-L_{t t}^{-1}\left(\left(u_{0}+\cdots+u_{n-1}\right)_{x x}\right), \quad n=1,2, \ldots
\end{array}
$$

Therefore, according to Eq.(23), we get:

$u(x, t)=\sin x\left(1-\frac{t^{2}}{2 !}+\frac{t^{4}}{4 !}-\frac{t^{6}}{6 !}+\cdots\right)$

This has the closed form

$u(x, t)=\sin x \cos t$

which is the exact solution of the problem and it is the same results obtained by VIM [3].

\subsection{Diffusion equations}

In this work we aim to investigate the following diffusion equations $[2,4,5]$ :

$u_{t}=u_{x x}+f(u), \quad 0<x<L, \quad t>0$,

$u_{t}=u_{x x}+g(x, t), \quad 0<x<L, \quad t>0$

$u_{t}=\left(D(u) u_{x}\right)_{x}, \quad 0<x<L, \quad t>0$,

The functions $f(u)$ and $g(x, t)$ are linear and source functions, respectively. $D(u)$ is the diffusion term, which plays an important role in wide range of applications in the diffusion processes. $D(u)$ appears in several functional forms, such as power law and exponential forms $[2,4,5]$. 


\subsubsection{The homogeneous linear diffusion equation}

We first consider the homogeneous linear diffusion equation [4]

$u_{t}=u_{x x}-u, 0<x<\pi, \quad t>0$,

with initial condition:

$u(x, 0)=\sin x$,

and the boundary conditions

$u(0, t)=0, \quad u(\pi, t)=0$.

The homogeneous equation (55) represents a heat equation with a lateral heat loss. This can be attributed to the additional term $-u(x, t)[4]$.

Eq.(55) can be written in an operator form as

$\tilde{L}_{t} u=u_{x x}-u$

where $\tilde{L}_{t}=\frac{\partial}{\partial t}$. Let us assume the the inverse operator $\tilde{L}_{t}^{-1}$ exists and it can be take with respect $t$ from 0 to $t$, i.e.

$\tilde{L}_{t}^{-1}()=.\int_{0}^{t}() d$.

Then, by taking the inverse operator $\tilde{L}_{t}^{-1}$ to both sides of the Eq.(55) and using the initial condition, leads to

$u(x, t)=\sin x+\tilde{L}_{t}^{-1}\left(u_{x x}-u\right)$.

By using the DJM, we achieve the following components:

$u_{0}(x, t)=\sin x$,

$u_{1}(x, t)=N\left(u_{0}\right)=\tilde{L}_{t}^{-1}\left(\left(u_{0}\right)_{x x}-u_{0}\right)=-2 t \sin x$,

$u_{2}(x, t)=N\left(u_{1}+u_{0}\right)-N\left(u_{0}\right)=\tilde{L}_{t}^{-1}\left(\left(u_{1}+u_{0}\right)_{x x}-\left(u_{1}+u_{0}\right)\right)-u_{1}=2 t^{2} \sin x$,

$$
\begin{array}{r}
u_{3}(x, t)=N\left(u_{2}+u_{1}+u_{0}\right)-N\left(u_{1}+u_{0}\right)=\tilde{L}_{t}^{-1}\left(\left(u_{2}+u_{1}+u_{0}\right)_{x x}-\left(u_{2}+u_{1}+u_{0}\right)\right)- \\
-\tilde{L}_{t}^{-1}\left(\left(u_{1}+u_{0}\right)_{x x}-\left(u_{1}+u_{0}\right)\right)=-\frac{4}{3} t^{3} \sin x,
\end{array}
$$

and so on. Therefore, according to Eq.(23), we get:

This has the closed form

$$
u_{n}(x, t)=\sin x\left(-(2 t)+\frac{(2 t)^{2}}{2 !}-\frac{(2 t)^{3}}{3 !}+\frac{(2 t)^{4}}{4 !}+\cdots\right)
$$

$u(x, t)=\sin x e^{-2 t}$.

which is the exact solution of the problem and it is the same results obtained by VIM [4]. 


\subsubsection{The inhomogeneous linear diffusion equation}

We consider the inhomogeneous linear diffusion equation [4]

$u_{t}=u_{x x}+\cos x, \quad 0<x<\pi, \quad t>0$,

with initial condition:

$u(x, 0)=0$,

and the boundary conditions

$u(0, t)=1-e^{-t}, \quad u(\pi, t)=-1+e^{-t}$.

According to the DJM, we achieve the following components:

$u_{0}(x, t)=t \cos x$

$u_{1}(x, t)=N\left(u_{0}\right)=\tilde{L}_{t}^{-1}\left(\left(u_{0}\right)_{x x}\right)=-\frac{1}{2} t^{2} \cos x$,

$u_{2}(x, t)=N\left(u_{1}+u_{0}\right)-N\left(u_{0}\right)=\tilde{L}_{t}^{-1}\left(\left(u_{1}+u_{0}\right)_{x x}\right)-u_{1}=\frac{1}{6} t^{3} \cos x$,

$$
\begin{array}{r}
u_{3}(x, t)=N\left(u_{2}+u_{1}+u_{0}\right)-N\left(u_{1}+u_{0}\right)=\tilde{L}_{t}^{-1}\left(\left(u_{2}+u_{1}+u_{0}\right)_{x x}\right)-L_{t t}^{-1}\left(\left(u_{1}+u_{0}\right)_{x x}\right)= \\
=-\frac{1}{24} t^{4} \cos x,
\end{array}
$$

and so on. Therefore, according to Eq.(23), we get:

$$
u_{n}(x, t)=\cos x\left(t-\frac{t^{2}}{2 !}+\frac{t^{3}}{3 !}-\frac{t^{4}}{4 !}+\cdots\right)
$$

This has the closed form

$u(x, t)=\cos x\left(1-e^{-t}\right)$.

which is the exact solution of the problem and it is the same results obtained by VIM [4].

\subsubsection{Fast diffusion processes}

This process is described by a diffusion term of the form $[2,4,5]$ :

$D(u)=u^{n}, \quad n<0$

For $n=-0.5$, Eq. (54) models the plasma diffusion and thermal expulsion of liquid helium $[2,4,5]$. For $n=-1$, Eq. (54) appears in the thermal limit approximation of Carlemans model of the Boltzman equation and the expansion into a vacuum of a thermalized electron cloud described by the isothermal Maxwellian distribution [2, 4, 5] and references therein. For $n=-2$, Eq. (54) is considered as a model of diffusion in high-polymeric systems. 
First we consider a fast diffusion process when $n=-1$, Eq.(54) becomes $[2,5]$ :

$u_{t}=\left(u^{-1} u_{x}\right)_{x}$,

subject to the initial condition of

$u(x, 0)=\frac{2 c}{(a+x)^{2}}$,

where $a$ and $c \neq 0$ are arbitrary constants.

By using the DJM, we achieve the following components:

$u_{0}(x, t)=\frac{2 c}{(a+x)^{2}}$,

$u_{1}(x, t)=N\left(u_{0}\right)=\tilde{L}_{t}^{-1}\left(u_{0}^{-1}\left(u_{0}\right)_{x}\right)_{x}=\frac{2 t}{(a+x)^{2}}$,

$u_{2}(x, t)=N\left(u_{1}+u_{0}\right)-N\left(u_{0}\right)=\tilde{L}_{t}^{-1}\left(\left(u_{1}+u_{0}\right)^{-1}\left(u_{1}+u_{0}\right)_{x}\right)_{x}-u_{1}=0$,

and so on. In fact, we have $u_{n}(x, t)=0$, for $n \geq 2$.

Therefore, according to Eq.(23), we get:

$u(x, t)=\frac{2(c+t)}{(a+x)^{2}}$.

which is the exact solution of the problem and it is the same results obtained by ADM [2], VIM and HPM in [5].

\subsubsection{Slow diffusion processes}

We next consider a slow diffusion process when $n=1$, Eq.(54) becomes $[2,5]$ :

$u_{t}=\left(u u_{x}\right)_{x}$,

subject to the initial condition of

$u(x, 0)=\frac{1}{c} x^{2} \quad x>0$,

where $c>0$ is an arbitrary constant.

According to the DJM, we achieve the following components:

$u_{0}(x, t)=\frac{1}{c} x^{2}$,

$u_{1}(x, t)=N\left(u_{0}\right)=\tilde{L}_{t}^{-1}\left(u_{0}\left(u_{0}\right)_{x}\right)_{x}=\frac{6}{c^{2}} t x^{2}$,

$u_{2}(x, t)=N\left(u_{1}+u_{0}\right)-N\left(u_{0}\right)=\tilde{L}_{t}^{-1}\left(\left(u_{1}+u_{0}\right)\left(u_{1}+u_{0}\right)_{x}\right)_{x}-u_{1}=\frac{36}{c^{3}} t^{2} x^{2}+\frac{72}{c^{4}} t^{3} x^{2}$, 


$$
\begin{array}{r}
u_{3}(x, t)=N\left(u_{2}+u_{1}+u_{0}\right)-N\left(u_{1}+u_{0}\right)=\tilde{L}_{t}^{-1}\left(\left(u_{2}+u_{1}+u_{0}\right)\left(u_{2}+u_{1}+u_{0}\right)_{x}\right)_{x}-\tilde{L}_{t}^{-1}\left(\left(u_{1}+u_{0}\right)\right. \\
\left.\left(u_{1}+u_{0}\right)_{x}\right)_{x}=\frac{144}{c^{4}} t^{3} x^{2}+\cdots,
\end{array}
$$

and so on. Therefore, according to Eq.(23), we get:

$$
u_{n}(x, t)=x^{2}\left(\frac{1}{c}+\frac{6 t}{c^{2}}+\frac{36 t^{2}}{c^{3}}+\frac{216 t^{3}}{c^{4}}+\cdots\right)
$$

This has the closed form

$u(x, t)=\frac{x^{2}}{c-6 t}$.

which is the exact solution of the problem and it is the same results obtained by ADM [2], VIM and HPM in [5].

\subsubsection{Other diffusion processes}

Other cases of diffusion processes when $D(u)=\frac{1}{1+u^{2}}$ or $D(u)=\frac{1}{u^{2}-1}$, Eq.(54) becomes $[2,5]$ :

$u_{t}=\left(\frac{1}{1+u^{2}} u_{x}\right)_{x}$

subject to the initial condition of

$u(x, 0)=\tan x, \quad x>0$.

Proceeding as before, we achieve the following components:

$u_{0}(x, t)=\tan x$,

$u_{1}(x, t)=N\left(u_{0}\right)=\tilde{L}_{t}^{-1}\left(\left(\frac{1}{1+u_{0}^{2}}\right)\left(u_{0}\right)_{x}\right)_{x}=0$,

$u_{2}(x, t)=N\left(u_{1}+u_{0}\right)-N\left(u_{0}\right)=\tilde{L}_{t}^{-1}\left(\left(\frac{1}{1+\left(u_{1}+u_{0}\right)^{2}}\right)\left(u_{1}+u_{0}\right)_{x}\right)_{x}-u_{1}=0$,

and so on. In fact, we have $u_{n}(x, t)=0$, for $n \geq 1$. Therefore, according to Eq.(23), we get:

$u(x, t)=\tan x$.

which is the exact solution of the problem and it is the same results obtained by ADM [2], VIM and HPM in [5].

When $D(u)=\frac{1}{u^{2}-1}$, Eq.(54) becomes $[2,5]$ :

$u_{t}=\left(\frac{1}{u^{2}-1} u_{x}\right)_{x}$,

subject to the initial condition of

$u(x, 0)=-\operatorname{coth} x, \quad x>0$. 
Proceeding as before, we achieve the following components:

$u_{0}(x, t)=-\operatorname{coth} x$

$u_{1}(x, t)=N\left(u_{0}\right)=\tilde{L}_{t}^{-1}\left(\left(\frac{1}{u_{0}^{2}-1}\right)\left(u_{0}\right)_{x}\right)_{x}=0$,

$u_{2}(x, t)=N\left(u_{1}+u_{0}\right)-N\left(u_{0}\right)=\tilde{L}_{t}^{-1}\left(\left(\frac{1}{\left(u_{1}+u_{0}\right)^{2}-1}\right)\left(u_{1}+u_{0}\right)_{x}\right)_{x}-u_{1}=0$,

and so on. In fact, we have $u_{n}(x, t)=0$, for $n \geq 1$. Therefore, according to Eq.(23), we get:

$u(x, t)=-\operatorname{coth} x$.

which is the exact solution of the problem and it is the same results obtained by ADM [2], VIM and HPM in [5].

It can also be clearly seen that the DJM is reliable and efficient for handling linear and nonlinear problems, homogeneous or inhomogeneous, and in a bounded domain or unbounded domain, in a straightforward manner. Unlike the Adomian decomposition method, where computational algorithms are normally used to deal with the nonlinear terms. No needs to calculate Lagrange multiplier in VIM or construct a homotopy and solve the corresponding algebraic equations in HPM. The DJM is used directly with no requirement or restrictive assumptions for the nonlinear terms.

\section{Conclusion}

In this paper, the reliable iterative method namely (NIM or DJM) is implemented to obtain the exact solutions for solving linear and nonlinear wave and diffusion equations using the initial condition only. The DJM is simple to understand and easy to implement and does not require any restrictive assumptions as required by some existing techniques. The obtained exact solution of applying the DJM is in full agreement with the results obtained with those methods available in the literature such as Adomian decomposition method [2], variational iteration method $[3,4,5]$, homotopy analysis method [4] and homotopy perturbation method [5]. The method gives rapid convergent and handles linear and nonlinear problems in a similar manner and can be easily comprehended with only a basic knowledge of Calculus. It is economical in terms of computer power/memory and does not involve tedious calculations. Moreover, by solving some examples, it is seems that the DJM appears to be very accurate to employ with reliable results.

\section{References}

[1] A.M. Wazwaz, Linear and Nonlinear Integral Equations Methods and Applications. Beijing and Springer-Verlag Berlin Heidelberg, (2011).

[2] A.M. Wazwaz, Exact solutions to nonlinear diffusion equations obtained by the decomposion method. Applied Mathematics and Computation 123 (2001) 109-122.

[3] A.M. Wazwaz, The variational iteration method: A reliable analytic tool for solving linear and nonlinear wave equations. Computers and Mathematics with Applications 54 (2007) 926-932.

[4] A.M. Wazwaz,The variational iteration method: A powerful scheme for handling linear and nonlinear diffusion equations. Computers and Mathematics with Applications 54 (2007) 933-939.

[5] A. Sadighi, D.D. Ganji, Exact solutions of nonlinear diffusion equations by variational iteration method. Computers and Mathematics with Applications 54 (2007) 1112-1121.

[6] V. Daftardar-Gejji, H. Jafari, An iterative method for solving nonlinear functional equations. Journal of Mathematical Analysis and Applications 316 (2006) 753-763. 
[7] V. Daftardar-Gejji, H. Jafari, Solving a System of Nonlinear Functional Equations Using Revised New Iterative Method. World Academy of Science, Engineering and Technology 68 (2012) 08-21.

[8] V. Daftardar-Gejji, H. Jafari, New iterative method: Application to partial differential equations. Applied Mathematics and Computation 203 (2008) 778-783.

[9] V. Daftardar-Gejji, H. Jafari, Solving fractional boundary value problems with Dirichlet boundary conditions using a new iterative method. Computers and Mathematics with Applications 59 (2010) 1801-180.

[10] M. Yaseen, M. Samraiz, S. Naheed, The DJ method for exact solutionsof Laplace equation. Results in Physics 3 (2013) $38-40$.

[11] V. Daftardar-Gejji, S. Bhalekar, An Iterative method for solving fractional differential equations. Proceedings in Applied Mathematics and Mechanics, 7(2007) 2050017-2050018.

[12] S. Bhalekar, V. Daftardar-Gejji, Solving a System of Nonlinear Functional Equations Using Revised New Iterative Method. World Academy of Science, Engineering and Technology, 68(2012) 806-810.

[13] M. Yaseen, M. Samraiz, The Modified New Iterative Method for Solving Linear and Nonlinear Klein-Gordon Equations. Applied Mathematical Sciences, 6(2012) 2979-2987.

[14] M. Yaghouti, H. Deilami, Numerical Solution of Singular Differential-Difference Equations. World Applied Programming, 3(2013) 182-189.

[15] I. Ullah, H. Khan and M. Rahim, Numerical Solutions of Higher Order Nonlinear Boundary Value Problems by New Iterative Method. Applied Mathematical Sciences, 7(2013) 2429-2439.

[16] A. Hemeda, M. Al-luhaibi, New iterative method for solving gas dynamic equation. International Journal of Applied Mathematical Research, 3(2014) 190-195.

[17] V. Daftardar-Gejji, H. Jafari, Convergence of the New Iterative Method. International Journal of Differential Equations, Article ID 989065, 10 pages. 